\title{
Theoretical progress on cusp effect and KI4 decays
}

\author{
Jürg Gasser*† \\ Inst. Theor. Physik \\ Universität Bern \\ Sidlerstrasse 5 \\ Ch-3012 Bern \\ E-mail: gasser@itp.unibe.ch
}

\begin{abstract}
The cusp effect in $K \rightarrow 3 \pi$ and data on $K_{e 4}$ decays allow one to extract experimental information on the elastic $\pi \pi$ scattering amplitude near threshold, and to confront the outcome of the analyses with predictions made in the framework of QCD. In my talk I pointed out that these predictions concern an isospin symmetric world, while experiments are carried out in the real world, where isospin breaking effects - generated by electromagnetic interactions and by the mass difference of the up and down quarks - are always present. I discussed the corrections required to account for these, so that a meaningful comparison with the predictions becomes possible. In particular, I pointed out that there is a spectacular isospin breaking effect in $K_{e 4}$ decays, overlooked so far. Once it is taken into account, the previous discrepancy between NA48/2 data on $K_{e 4}$ decays and the prediction of $\pi \pi$ scattering lengths disappears.
\end{abstract}

Kaon International Conference

May 21-25, 2007

Laboratori Nazionali di Frascati dell'INFN

\footnotetext{
* Speaker.

'Work supported by the Swiss National Science Foundation, and by the EU contract N0. MRTN-CT-2006035482,"FLAVIAnet".
} 


\section{Introduction}

Chiral perturbation theory (ChPT) [1], combined with Roy equations, allows one to make very precise predictions for the values of the threshold parameters in elastic $\pi \pi$ scattering [2] - see Colangelo's contribution to these proceedings for a status report [3]. Several experiments allow one to confront these predictions with the data: i) $K^{+} \rightarrow \pi^{+} \pi^{-} e^{+} v_{e}$ decays $[4,5,6]$, ii) the pionium lifetime, measured by the DIRAC collaboration [7], and iii) the cusp effect in $K \rightarrow 3 \pi$ decays, investigated by the NA48/2 collaboration [6].

The experiments performed by the NA48/2 collaboration have generated an impressive data basis, as a result of which the matrix elements of $K_{e 4}$ and $K \rightarrow 3 \pi$ decays can be determined with an unprecedented accuracy. The interpretation of these measurements was the topic of my talk. In particular, I pointed out that the theoretical predictions and the measurements are performed in two different settings: the predictions concern pure QCD, in the isospin symmetry limit $m_{u}=m_{d}$, with photons absent. To be more precise, the convention is to choose the quark masses and the renormalization group invariant scale of QCD such that the pion and the kaon masses coincide with the values of the charged ones, and the pion decay constant is $F_{\pi}=92.4 \mathrm{MeV}$. [I do not specify the masses of the heavy quarks, because in the present context, their precise values do not matter.] I refer to this framework as a paradise world.

On the other hand, experiments are all carried out in the presence of isospin breaking effects, generated by real and virtual photons, and by the mass difference of the up and down quarks: this is the real world, described by the Standard Model. We are thus faced with the problem to find the relation between quantities measured in the real world, where isospin breaking effects are always present, and the predictions made in the paradise world.

Remarks: The situation is quite involved: 1 . The above mentioned three processes do not take place in the paradise world: the kaon is stable, whereas pionium cannot form, because electromagnetic interactions are absent. 2. In the real world, the processes i) and iii) do not occur either, because decays that involve external charged particles have zero probability to happen without the emission of photons. 3. Lattice calculations are not confronted with this problem: the evaluation of e.g. $\pi \pi$ or $\pi K$ scattering lengths [8] can be performed directly in the framework of pure QCD, at $m_{u}=m_{d}$ : isospin breaking effects can be excluded by fiat. I refer the interested reader to Colangelo's contribution [3] , and to Ref. [9] for a short review of lattice results concerning $\pi \pi$ scattering lengths.

\section{The cusp in $K \rightarrow 3 \pi$}

Cabibbo and Isidori pointed out [10] that cusp effects in the decay $K^{+} \rightarrow \pi^{+} \pi^{0} \pi^{0}$ may allow one to measure $S$-wave $\pi \pi$ scattering lengths, and thus to check the precise predictions made in the framework of chiral perturbation theory, see also [11]. A first data analysis based on this observation has appeared [12] already some time ago, and the latest developments were discussed at this conference [6].

We have started to investigate this process in the framework of non-relativistic quantum field theory in Ref. [13]. The status of our calculation is as follows. 
- Our amplitude agrees with the one of $[10,11]$ near the cusp, whereas it differs away from it. The NA48/2 collaboration at CERN has analyzed data with our amplitude as well. The outcome for the scattering length combination $a_{0}-a_{2}$ is in agreement [14] with the use of the amplitude from [10].

- We have evaluated the decay amplitude for the neutral kaon, $K_{L}^{0} \rightarrow \pi^{+} \pi^{-} \pi^{0}, \pi^{0} \pi^{0} \pi^{0}$, have written a pertinent FORTRAN code for the decay probability and made it available to the NA48/2 collaboration [15]. In the channel with 3 neutral pions, a cusp occurs as well. In this case, its strength is suppressed [6]. Nevertheless, the huge amount of data available at NA48/2 should make it possible to extract the same combination of scattering lengths as well, although with less precision [6].

- It remains to calculate electromagnetic corrections to these decays. Because we make use of a quantum field theory framework, our method allows us to perform these calculations in a straightforward, yet tedious manner. We have investigated the role of the formation of pionium near the cusp and have identified an interval around the cusp, where radiative corrections can be calculated reliably, in a systematic manner [15]. [In Ref. [16], the structure of the cusp in the presence of re-summed Coulomb ladders is investigated as well. In case that the same approximation is made, the so induced modifications of the decay distribution agree in the two frameworks. Furthermore, pionium production was calculated in [17], and compared with data by the NA48/2 collaboration [6].]

- Presently, we investigate the emission of real photons [15]. We evaluate the infrared divergent contributions in $K \rightarrow 3 \pi(\gamma)$ in dimensional regularization, and organize the remaining integrations over phase space in such a manner that the calculations can be performed numerically reasonably fast, so that a corresponding FORTRAN code can be used by the NA48/2 collaboration for performing fits.

\section{3. $K_{e 4}$ decays}

\subsection{General}

In the NA48/2 experiment, the general purpose Monte Carlo program PHOTOS [18] is used to calculate electromagnetic corrections. In addition, the Sommerfeld factor is applied, to account for the Coulomb interaction between charged particles [19].

In my talk, I pointed out that in these prescriptions to perform radiative corrections, one specific mechanism is not included. Namely, the kaon may decay first into a neutral pion pair, that then annihilates into two charged pions, or first decay into a charged pion pair, that then re-scatters. In the real world, the neutral pion mass is smaller than the charged one by about $4.6 \mathrm{MeV}^{1}$. As a result of this, the two contributions to the decay matrix element have a different holomorphic structure: the neutral (charged) pion loop generates a branch point at $s_{\pi}=4 M_{\pi^{0}}^{2}$ (at $s=4 M_{\pi}^{2}$ ), and the phase of the relevant form factor is affected with a cusp, and does not vanish at the threshold $s=4 M_{\pi}^{2}$. In my talk, I discussed the case of the scalar form factor $\left\langle\pi^{+} \pi^{-}|\bar{u} u+\bar{d} d| 0\right\rangle$, where the same effect

\footnotetext{
${ }^{1}$ Throughout the text, I use the symbols $M_{\pi}\left(M_{\pi^{0}}\right)$ for the charged (neutral) pion mass.
} 
occurs. Assuming universality, I applied the result to $K_{e 4}$ decays [20]. Meanwhile, we have worked out [21] the effect for $K_{e 4}$ decays as well. I describe the outcome of the calculation shortly here, and refer the interested reader to our forthcoming publication for details [21].

\subsection{Partial wave expansion: isospin symmetry limit}

The matrix element for $K^{+}(p) \rightarrow \pi^{+}\left(p_{1}\right) \pi^{-}\left(p_{2}\right) e^{+}\left(p_{e}\right) v_{e}\left(p_{v}\right)$ is

$$
T=\frac{G_{F}}{\sqrt{2}} V_{u s}^{\star} \bar{u}\left(p_{v}\right) \gamma^{\mu}\left(1-\gamma_{5}\right) v\left(p_{e}\right)\left(V_{\mu}-A_{\mu}\right),
$$

where the last factor denotes hadronic matrix elements of the strangeness changing (vector and axial vector) currents,

$$
V_{\mu}-A_{\mu}=\left\langle\pi^{+}\left(p_{1}\right) \pi^{-}\left(p_{2}\right) \text { out }\left|\left(\bar{s} \gamma_{\mu} u-\bar{s} \gamma_{\mu} \gamma_{5} u\right)\right| K^{+}(p)\right\rangle .
$$

In the following, I concentrate on the matrix element of the axial vector current, because it carries information on the $\pi \pi$ final state interactions and, in particular, on the $\pi \pi$ phases. One decomposes $A_{\mu}$ into Lorentz scalars,

$$
A_{\mu}=-i \frac{1}{M_{K}}\left[\left(p_{1}+p_{2}\right)_{\mu} F+\left(p_{1}-p_{2}\right)_{\mu} G+\left(p_{e}+p_{v}\right)_{\mu} K\right] .
$$

The form factors $F, G, K$ are holomorphic functions of the three variables

$$
s_{\pi}=\left(p_{1}+p_{2}\right)^{2}, t=\left(p_{1}-p\right)^{2}, u=\left(p_{2}-p\right)^{2} .
$$

Sometimes, it is useful to use instead

$$
s_{\pi}=\left(p_{1}+p_{2}\right)^{2}, s_{\ell}=\left(p_{e}+p_{v}\right)^{2}, \cos \theta_{\pi},
$$

where $\theta_{\pi}$ is the angle of the $\pi^{+}$in the CM system of the two charged pions, with respect to the dipion line of flight in the rest system of the kaon $[22,5]$. In the isospin symmetry limit, one identifies the $\pi \pi$ phases in the matrix element in a standard manner, by performing a partial wave expansion, and using unitarity and analyticity, although, in the present case, this is a slightly intricate endeavor [23]. It is useful to introduce a particular combination of form factors,

$$
F_{1}=F+\frac{\left(M_{K}^{2}-s_{\pi}-s_{\ell}\right) \sigma}{\lambda\left(M_{K}^{2}, s_{\pi}, s_{\ell}\right)^{1 / 2}} \cos \theta_{\pi} G
$$

Here, $\sigma=\sqrt{1-4 M_{\pi}^{2} / s_{\pi}}$, and $\lambda(x, y, z)$ is the triangle function. The form factor $F_{1}$ has a simple partial wave expansion,

$$
F_{1}=f\left(s_{\pi}, s_{\ell}\right)+\sum_{k \geq 1} P_{k}\left(\cos \theta_{\pi}\right) f_{k}\left(s_{\pi}, s_{\ell}\right)
$$

In the low-energy region $s_{\pi} \leq 16 M_{\pi}^{2}, f_{k}$ carry the $\pi \pi$ phases [23] in the pertinent isospin channel. In the following, I consider the lowest partial wave $f\left(s_{\pi}, s_{\ell}\right)$. In the interval $4 M_{\pi}^{2} \leq s_{\pi} \leq 16 M_{\pi}^{2}$, its phase coincides with the isospin zero $S$-wave phase $\delta_{0}^{0}$ in elastic $\pi \pi$ scattering,

$$
f_{+}=e^{2 i \delta_{0}^{0}} f_{-}, f_{ \pm}=f\left(s_{\pi} \pm i \varepsilon, s_{\ell}\right) .
$$




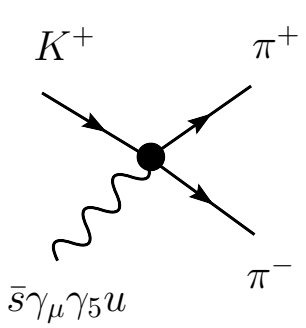

a)

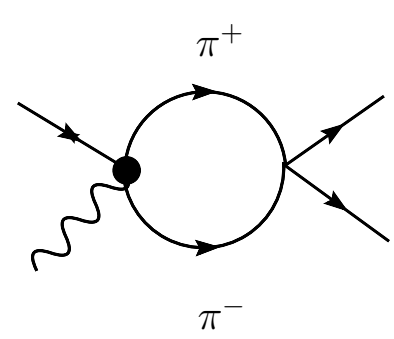

b)

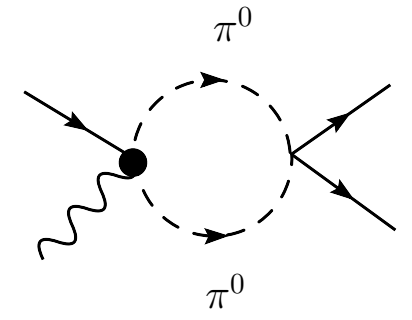

c)

Figure 1: Some of the graphs that contribute to the matrix element of the axial current at tree and oneloop order. The filled vertex indicates that the axial current also couples to a single kaon line. That graph contributes to the form factor $K$. There are many additional graphs at one-loop order, not displayed in the figure.

It is instructive to calculate the form factors in chiral perturbation theory and to verify that $F_{1}$ indeed has the behaviour just discussed. For this, it is sufficient to consider the effective Lagrangian

$$
\mathscr{L}_{2}=\frac{F_{0}^{2}}{4}\left\langle D_{\mu} U D^{\mu} U^{\dagger}+2 B_{0} \mathscr{M}\left(U+U^{\dagger}\right)\right\rangle,
$$

where the covariant derivative $D_{\mu} U$ contains the external vector and axial vector currents, and $\mathscr{M}=\operatorname{diag}\left(\hat{m}, \hat{m}, m_{s}\right)$. Some of the graphs that contribute at tree level and at one loop are displayed in Figure 1. The result is [24]

$$
f\left(s_{\pi}, s_{\ell}\right)=\frac{M_{K}}{\sqrt{2} F_{0}}\left\{1+\Delta\left(s_{\pi}\right)+H\left(s_{\pi}, s_{\ell}\right)+O\left(p^{4}\right)\right\}
$$

with

$$
\begin{aligned}
\Delta\left(s_{\pi}\right) & =\frac{1}{2 F_{0}^{2}}\left(2 s_{\pi}-M_{\pi}^{2}\right) \bar{J}\left(s_{\pi}\right), \\
16 \pi^{2} \bar{J}\left(s_{\pi}\right) & =\sigma\left(\ln \frac{1-\sigma}{1+\sigma}+i \pi\right)+2, \quad s_{\pi} \geq 4 M_{\pi}^{2} .
\end{aligned}
$$

Here, $M_{\pi}\left(F_{0}\right)$ denotes the pion mass (pion decay constant), at leading order in the chiral expansion. The quantity $H\left(s_{\pi}, s_{\ell}\right)$ is real in the interval of elastic $\pi \pi$ scattering. It is now seen that $f$ indeed has the property (3.1) at this order in the low-energy expansion, with

$$
\delta_{0}^{0}=\frac{\left(2 s_{\pi}-M_{\pi}^{2}\right)}{32 \pi F_{0}^{2}} \sigma .
$$

This is the phase of the isospin zero $S$-wave, in tree approximation.

\subsection{Partial wave expansion: the real world}

In reality, experiments are not carried out in the paradise world just discussed: we have not included so far photons, nor did we consider isospin breaking effects generated by different up and down quark masses. Here, I investigate these effects in several steps: 


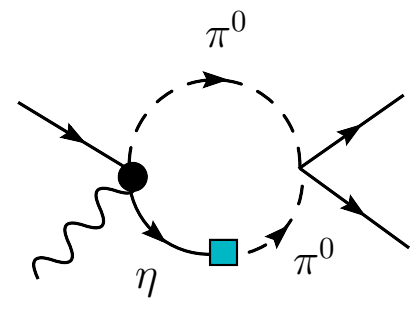

Figure 2: The contribution from $\pi^{0}-\eta$ mixing, at leading order in $m_{d}-m_{u}$. The filled square denotes the vertex from $\pi^{0}-\eta$ mixing. Otherwise, the notation is the same as in Figure 1.

i) I assume that the manner in which real and virtual photons are treated in the analysis of the NA48/2 experiment (PHOTOS + Sommerfeld factor) is a decent approximation to the effects generated by soft photons.

ii) This procedure misses the effects generated by the pion and kaon mass differences, and by the quark mass difference $m_{d}-m_{u}$. These must therefore be taken into account separately.

iii) $\mathrm{ChPT}$ is the appropriate tool to evaluate these contributions.

iv) I assume that PHOTOS+Sommerfeld factor + mass effects provide a good approximation to the full isospin breaking contributions.

Remark: One may envisage a more ambitious procedure [25], by working out the relevant matrix elements in the framework of ChPT including photons and leptons [26], and then constructing a new event generator, to be used in the analysis of $K_{e 4}$ decays. [A one-loop calculation was already performed in [27]. It needs to be checked, and brought into a form which is suitable for the present purpose.] Eventually, such an analysis might lead to an improved algorithm, but I consider it a long term project. End of remark.

According to iii), we simply need to perform a ChPT calculation of the effects generated by the mass differences. This is rather easy to achieve at one-loop order: one adapts the quark mass matrix, $\mathscr{M} \rightarrow \operatorname{diag}\left(m_{u}, m_{d}, m_{s}\right)$, and enlarges the Lagrangian $\mathscr{L}_{2}$ [28],

$$
\mathscr{L}_{2} \rightarrow \mathscr{L}_{2}+C\left\langle Q U Q U^{\dagger}\right\rangle, Q=\frac{e}{3} \operatorname{diag}(2,-1,-1),
$$

where $C$ is a new low-energy constant, that breaks the isospin symmetry of the meson masses: $M_{\pi} \neq M_{\pi^{0}}, M_{K} \neq M_{K^{0}}$.

The effect of the replacement (3.3) is twofold: first, as just mentioned, the meson masses split. As a result of this, the loop contributions in Fig. 1b),c) have a different threshold, and the phase of the form factor $f$ generates a cusp. Second, in addition to the graphs displayed in Figure 1, there is a new contribution shown in Figure 2: the kaon interacts with the axial current to generate a $\pi^{0} \eta$ intermediate state. Because $m_{u} \neq m_{d}$, the $\eta$ can transform back into a neutral pion, that then re-scatters with the second neutral pion into a charged pion pair.

Working out the relevant diagrams, one finds that the phase (3.2) becomes in the elastic region

$$
\delta_{0}^{0} \rightarrow \delta=\frac{1}{32 \pi F_{0}^{2}}\left\{\left(4 \Delta_{\pi}+s_{\pi}\right) \sigma+\left(s_{\pi}-M_{\pi^{0}}^{2}\right)\left(1+\frac{3}{2 R}\right) \sigma_{0}\right\}
$$


with

$$
\Delta_{\pi}=M_{\pi}^{2}-M_{\pi^{0}}^{2}, \quad \sigma_{0}=\sqrt{1-4 M_{\pi^{0}}^{2} / s_{\pi}}, \quad R=\frac{m_{s}-\hat{m}}{m_{d}-m_{u}} .
$$

The one-loop expressions for the form factors $F, G$ given in Refs. [27] contain the effects considered here, up to terms of order $\alpha_{Q E D}\left(m_{d}-m_{u}\right)$.

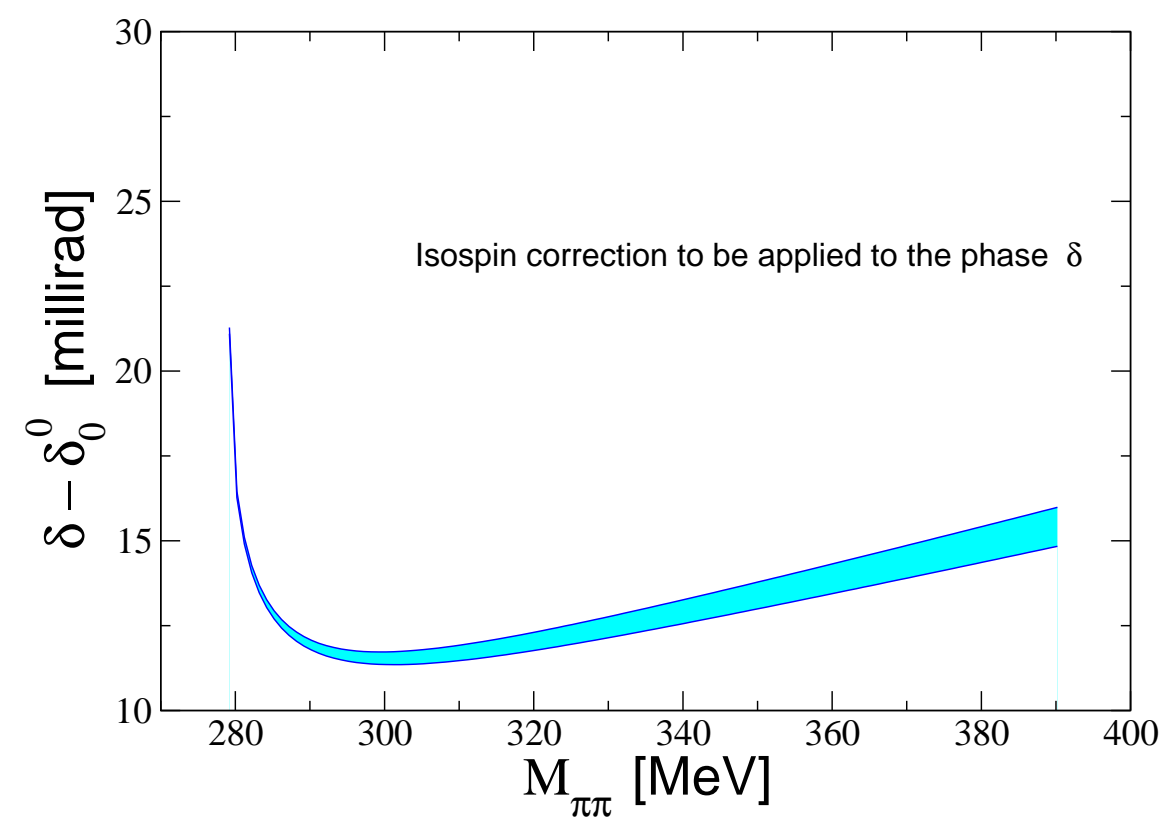

Figure 3: The isospin breaking correction that must be subtracted from the phase $\delta$ measured in $K_{e 4}$ decays. The width of the band reflects the uncertainty in the ratio $R$.

I consider the result (3.4) to be very interesting, for the following reasons. First, due to the presence of the phase space factor $\sigma_{0}$, the phase $\delta$ does not vanish at the threshold $s_{\pi}=4 M_{\pi}^{2}$. This unexpected behaviour of the phase is the cusp effect already experienced in $K \rightarrow 3 \pi$ decays, with the role of neutral and charged pions interchanged. Second, the difference $\delta-\delta_{0}^{0}$ is positive for $s_{\pi}$ above the threshold, and even increases at large $s_{\pi}$,

$$
\delta-\delta_{0}^{0}=\frac{3 s_{\pi}}{64 \pi F_{0}^{2}} \frac{1}{R}+O(1), \quad s_{\pi} / M_{\pi}^{2} \gg 1 .
$$

We now come to the main point. According to point iv) above, it is the phase $\delta$ that is measured in $K_{e 4}$ decays (up to contributions from higher orders in the chiral expansion). Therefore, before comparing the phase so determined with ChPT predictions, one has to subtract from the measured phase the (positive) difference $\delta-\delta_{0}^{0}$, because $\delta_{0}^{0}=\delta-\left(\delta-\delta_{0}^{0}\right)$. In Figure 3 we display this difference in the relevant decay region, for $R=37 \pm 4^{2}$. The width of the band reflects the uncertainty

\footnotetext{
${ }^{2}$ This value for $R$ should be considered as preliminary - it was used in my talk for illustrative purposes. A more refined estimate will be provided in [21]. Of course, the conclusions to be drawn from the isospin breaking effects considered here will not change.
} 
in $R$. [As I pointed out in my talk, two-loop contributions are modest in the analogous case of the scalar form factor of the pion [21].] It is seen that the isospin correction is quite substantial - well above the uncertainties quoted for the measured phase [5]. [In Ref. [29], the cusp in $K_{e 4}$ decays was investigated as well. The expressions presented there do not agree with (3.4), because these authors do not take into account derivative couplings of the $\pi \pi$ amplitude, as is dictated by chiral symmetry.]

Colangelo has performed fits to $K_{e 4}$ data, with and without isospin breaking corrections applied. It turns out that the former discrepancy [30] of the NA48/2 data with the prediction [2] disappears, once isospin breaking effects are taken into account in the manner just described, see [3]. Colangelo also shows that the former agreement between the chiral prediction and the E865 data [4] becomes marginal. This is an issue that should be understood, because it is independent of the special effects considered here. On the other hand, since the NA48/2 data are so precise, they will dominate the E865 result, in any case.

Note added: Isidori has meanwhile worked out soft photon corrections for $K^{+} \rightarrow \pi^{+} \pi^{+} \pi^{-}$ [31].

\section{Acknowledgments}

It is a pleasure to thank the organizers for the invitation to give this talk, and for the excellent organization of this exciting conference at that beautiful place. I thank M. Bissegger, G. Colangelo, A. Fuhrer, B. Kubis, H. Leutwyler and A. Rusetsky for a most enjoyable collaboration, B. BlochDevaux, L. DiLella and I. Mannelli for explaining to me with patience details of the data analysis. I am grateful to M. Knecht for discussions, and A.V. Tarasov for communications concerning topics considered here. Finally, I thank G. Colangelo, H. Leutwyler, J. R. Peláez, A. Rusetsky and F. J. Ynduráin for useful comments on the manuscript.

\section{References}

[1] S. Weinberg, Physica A 96 (1979) 327.

[2] G. Colangelo, J. Gasser and H. Leutwyler, Nucl. Phys. B 603 (2001) 125 [arXiv:hep-ph/0103088].

[3] G. Colangelo, contribution to this conference [arXiv:0710.3050 [hep-ph]].

[4] L. Rosselet et al., Phys. Rev. D 15 (1977) 574;

S. Pislak et al. [BNL-E865 Collaboration], Phys. Rev. Lett. 87 (2001) 221801 [arXiv:hep-ex/0106071];

S. Pislak et al. [BNL-E865 Collaboration], Phys. Rev. D 67 (2003) 072004 [arXiv:hep-ex/0301040].

[5] B. Bloch-Devaux, contribution to this conference.

[6] L. DiLella, contribution to this conference;

E. Goudzovski, contribution to this conference.

[7] L. Tauscher, contribution to this conference.

[8] T. Yamazaki et al. [CP-PACS Collaboration], Phys. Rev. D 70 (2004) 074513 [arXiv:hep-lat/0402025]; 
S. R. Beane, P. F. Bedaque, K. Orginos and M. J. Savage [NPLQCD Collaboration], Phys. Rev. D 73 (2006) 054503 [arXiv:hep-lat/0506013];

S. R. Beane, P. F. Bedaque, T. C. Luu, K. Orginos, E. Pallante, A. Parreno and M. J. Savage, Phys. Rev. D 74 (2006) 114503 [arXiv:hep-lat/0607036];

S. R. Beane, T. C. Luu, K. Orginos, A. Parreno, M. J. Savage, A. Torok and A. Walker-Loud, arXiv:0706.3026 [hep-lat].

[9] H. Leutwyler, Insights and puzzles in light quark physics, talk given at: 42nd Rencontres de Moriond on QCD and Hadronic Interactions, La Thuile, Italy, 17-24 March 2007, to appear in the proceedings, arXiv:0706.3138 [hep-ph].

[10] N. Cabibbo, Phys. Rev. Lett. 93 (2004) 121801 [arXiv:hep-ph/0405001];

N. Cabibbo and G. Isidori, JHEP 0503 (2005) 021 [arXiv:hep-ph/0502130].

[11] E. Gamiz, J. Prades and I. Scimemi, Eur. Phys. J. C 50 (2007) 405 [arXiv:hep-ph/0602023].

[12] J. R. Batley et al. [NA48/2 Collaboration], Phys. Lett. B 633, 173 (2006) [arXiv:hep-ex/0511056].

[13] G. Colangelo, J. Gasser, B. Kubis and A. Rusetsky, Phys. Lett. B 638 (2006) 187 [arXiv:hep-ph/0604084].

[14] NA48/2 collaboration, private communication.

[15] J. Gasser, M. Bissegger, A. Fuhrer, B. Kubis and A. Rusetsky, work in progress.

[16] S. R. Gevorkyan, A. V. Tarasov and O. O. Voskresenskaya, Phys. Lett. B 649 (2007) 159;

S. R. Gevorkyan, D. T. Madigozhin, A. V. Tarasov and O. O. Voskresenskaya, arXiv:hep-ph/0702154.

[17] Z. K. Silagadze, JETP Lett. 60 (1994) 689 [arXiv:hep-ph/9411382].

[18] E. Barberio and Z. Was, Comput. Phys. Commun. 79 (1994) 291;

E. Barberio, B. van Eijk and Z. Was, Comput. Phys. Commun. 66 (1991) 115.

[19] B. Bloch-Devaux, private communication.

[20] J. Gasser and A. Rusetsky, internal note, March 2007.

[21] G. Colangelo, J. Gasser and A. Rusetsky, to appear.

[22] N. Cabibbo and A. Maksymovicz, Phys. Rev. 137 (1965) B438; erratum Phys. Rev. 168 (1968) 1926.

[23] F. A. Berends, A. Donnachie and G. C. Oades, Phys. Lett. 26B (1967) 109; Phys. Rev. 171 (1968) 1457.

[24] J. Bijnens, Nucl. Phys. B 337 (1990) 635 ;

C. Riggenbach, J. Gasser, J. F. Donoghue and B. R. Holstein, Phys. Rev. D 43 (1991) 127.

[25] M. Knecht, private communication, and internal note, June 2007.

[26] M. Knecht, H. Neufeld, H. Rupertsberger and P. Talavera, Eur. Phys. J. C 12 (2000) 469 [arXiv:hep-ph/9909284].

[27] V. Cuplov and A. Nehme, arXiv:hep-ph/0311274;

A. Nehme, Phys. Rev. D 69 (2004) 094012 [arXiv:hep-ph/0402007];

A. Nehme, Eur. Phys. J. C 40 (2005) 367 [arXiv:hep-ph/0408104].

[28] R. Urech, Nucl. Phys. B 433 (1995) 234 [arXiv:hep-ph/9405341];

H. Neufeld and H. Rupertsberger, Z. Phys. C 71 (1996) 131 [arXiv:hep-ph/9506448]. 
[29] S. R. Gevorkyan, A. N. Sissakian, A. V. Tarasov, H. T. Torosyan and O. O. Voskresenskaya, arXiv:0704.2675 [hep-ph].

[30] B. Bloch-Devaux, Recent results from NA48/2 on $K_{e 4}$ and $K_{3 \pi}$ decays - Interpretation in terms of $\pi \pi$ scattering lengths, talk given at: QCD 2006, Montpellier, France, 3-7 July 2006, to appear in the proceedings;

B. Bloch-Devaux, Recent results from $N A 48 / 2$ on $K_{e 4}$ and $K_{3 \pi}$ decays - Interpretation in terms of $\pi \pi$ scattering lengths, talk given at: XLIInd Rencontres de Moriond QCD 2007, La Thuile, Italy, 17-24 March 2007, to appear in the proceedings.

[31] G. Isidori, arXiv:0709.2439 [hep-ph]. 